\title{
Apoptosis and proliferation in Staphylococcus aureus-challenged, nonlactating mammary glands stimulated to grow rapidly and develop with estradiol and progesterone
}

\author{
B. D. Enger, ${ }^{1}$ S. C. Nickerson, ${ }^{2}$ H. L. M. Tucker, ${ }^{1}$ C. L. M. Parsons, ${ }^{1}$ and R. M. Akers ${ }^{1 *}$ \\ ${ }^{1}$ Dairy Science Department, Virginia Polytechnic Institute and State University, Blacksburg 24060 \\ ${ }^{2}$ Animal and Dairy Science Department, University of Georgia, Athens 30602
}

\section{ABSTRACT}

Bovine mastitis is a common and costly disease in the dairy industry and is known to negatively affect the amount of epithelium in nonlactating mammary glands. Despite this recognition, an understanding of the mechanisms contributing to reductions in epithelium is lacking. The objective of this study was to evaluate cellular apoptosis and proliferation in uninfected and Staphylococcus aureus-infected mammary glands that were stimulated to rapidly grow and develop. Estradiol and progesterone injections were administered to 18 nonlactating dairy cows to induce mammary growth, and 2 quarters from each animal were infused with saline or Staph. aureus. Mammary tissues were collected at $5(\mathrm{n}=9)$ and $10 \mathrm{~d}(\mathrm{n}=9)$ postinfusion and examined using quantitative bright field and florescent immunohistochemistry. Staphylococcus aureus mammary glands tended to have a greater number of mammary epithelial cells undergoing apoptosis than saline quarters. In the stromal compartment, challenged quarters contained a lower proportion of cells undergoing apoptosis than saline quarters overall; however, cell types undergoing apoptosis were differentially affected. Staphylococcus aureus quarters contained a lesser percentage of apoptotic fibroblasts while also containing more nonapoptotic immune cells than saline quarters in the intralobular stroma compartment. A similar number of proliferating epithelial cells were present in Staph. aureus and saline mammary tissues, but more proliferating cells were present in the intralobular stroma compartment of Staph. aureus-infused quarters than those infused with saline. When these cellular responses are considered together, it indicates that changes in cel-

Received August 3, 2018.

Accepted September 24, 2018.

*Corresponding author: rma@vt.edu lular apoptosis and proliferation contribute to changes in the gland structure by potentiating the expansion of the intralobular stromal compartment, via cellular accumulation, and limiting the amount of epithelium due to increases in cellular apoptosis in affected glands. Reductions in mammary epithelium are expected to reduce future milk yields and productive herd life.

Key words: mastitis, dry cow, heifer, mammary growth, intramammary infection

\section{INTRODUCTION}

Bovine mastitis is almost exclusively the result of a bacterial IMI and continues to be a common and challenging disease for US and global dairy industries. It is well recognized that mastitis in lactating mammary glands negatively affects the synthesis and secretion of milk components as well as mammary structure. For instance, reductions in milk yield and quality occur as milk SCC (a measure of mammary inflammation) increases (Jones et al., 1984; Hogan et al., 2016), and many investigators have previously described (Spencer, 1949; Nickerson, 1980; Akers and Thompson, 1987) and reviewed (Akers and Nickerson, 2011) the marked infiltration of immune cells, fibrosis of affected tissues, and atrophy of secretory tissues relative to nonmastitic glands that result in response to mastitis.

Milk yield is a function of the number of secretory mammary epithelial cells in the mammary gland and their secretory activity; a reduction of either would negatively affect milk yield. In relation to mastitis, it is expected that the pathogens and inflammation implicated in this disease create conditions that would reduce the cell numbers in the gland via apoptosis and, potentially, necrosis during acute infections. Previously, Bayles et al. (1998) observed apoptosis of MAC-T cells (a bovine mammary epithelial cell line) when cells were challenged with Staphylococcus aureus. Hu et al. (2014) also challenged bovine mammary epithelial cells in vitro with Staph. aureus and described activation of the 
Fas-FADD apoptosis pathway and increased activities of the integral apoptosis enzymes caspase-3 and caspase-8. Expanding this work into the lactating bovine mammary gland, Long et al. (2001) established that intramammary infusion of Escherichia coli also induces apoptosis of mammary epithelial cells. Undoubtedly, induced death of mammary epithelial cells when the mammary gland is growing, which would eventually give rise to secretory epithelium and functional alveoli, would be undesirable and would likely negatively affect future milk yields.

Aside from IMI in lactating animals, IMI are common in nonlactating heifers (Fox, 2009) and dry cows (Eberhart, 1986). The occurrence of IMI in these animals is concerning given the considerable amount of mammary growth that occurs during first gestation (Tucker, 1987) and the last half of the dry period (Capuco et al., 1997). The effects of IMI in the mammary glands of nonpregnant, nulliparous heifers have been previously investigated, and reductions in mammary epithelium and increased stromal tissue areas were described (Trinidad et al., 1990). More recently, Andreotti et al. (2017) investigated the effects of Staph. aureus IMI in cows during the first $3 \mathrm{wk}$ of the dry period and observed an increased percentage of epithelial cells undergoing apoptosis in Staph. aureus-infected quarters relative to uninfected quarters. When these reports are taken together, it is evident that IMI in nonlactating mammary glands negatively affects mammary gland structure by reducing mammary epithelium and increasing the percentage of mammary epithelial cells undergoing apoptosis, which is expected to reduce future milk yields. Although the results of these 2 reports (Trinidad et al., 1990; Andreotti et al., 2017) are telling, neither investigates how the mammary gland is affected by IMI during periods of rapid mammary growth and development, such as in the pregnant dairy heifer or during the last half of the dry period in the cow.

Previously, Enger et al. (2018) examined Staph. aureus IMI effects on mammary tissue structure in glands that were stimulated to rapidly grow and develop, and observed a significant reduction in intralobular areas occupied by epithelium and increased areas of intralobular stroma. It is unknown what chief mechanisms contribute to these changes in tissue structure, but a greater number of mammary epithelial cells would be undergoing apoptosis in challenged glands based on prior studies. The objective of the current study was to evaluate cellular apoptosis and proliferation, using quantitative histology, in uninfected and Staph. aureusinfected mammary glands that were stimulated to rap- idly grow and develop using estradiol and progesterone injections.

\section{MATERIALS AND METHODS}

\section{Study Design}

The mammary tissues we used were collected as described in detail by Enger et al. (2018). In brief, 19 nonpregnant, nonlactating dairy cows that had been dry for $45 \mathrm{~d}$ were administered 7 consecutive daily injections of estradiol $(0.1 \mathrm{mg} / \mathrm{kg}$ of $\mathrm{BW})$ and progesterone $(0.25 \mathrm{mg} / \mathrm{kg}$ of BW) to stimulate mammary growth and development. Three days after the last injection, 2 culture-negative quarters of each cow were randomly infused with either sterile, physiological PBS ( $\mathrm{n}=19)$ or Staph. aureus $(\mathrm{n}=19)$. The Staph. aureus Novel strain (Smith et al., 1998) was used as the challenge organism and infused at a target inoculum of 5,000 cfu. All saline-infused quarters remained culture negative throughout the trial, and all but 1 cow developed a Staph. aureus IMI in the challenged quarter. The quarters that developed Staph. aureus IMI displayed limited signs of clinical infection, as no localized inflammation was visually apparent and glands did not feel abnormal, but small flakes could occasionally be observed in the collected mammary secretions. At 5 d postchallenge, half of the cows were randomly selected for euthanasia and tissue collection; tissues were collected from the remaining animals at $10 \mathrm{~d}$ postchallenge. Mammary parenchyma that was most proximal to the teat, but dorsal to the gland cistern, was collected from experimental quarters. Tissues were fixed and processed as previously described by Enger et al. (2018). The mammary tissues collected from the animal that did not establish a Staph. aureus IMI were not used. This work was approved by the Virginia Polytechnic Institute and State University Institutional Animal Care and Use Committee (protocol \#15-196).

\section{Immunohistochemistry}

Two mammary tissue slide sets were prepared: one was used to examine expression of cleaved caspase-3 (an apoptosis marker) and the other used to examine expression of Ki-67 (a proliferation marker) in the collected mammary tissues. Tissues were sectioned $5-\mu \mathrm{m}$ thick using a rotary microtome (model HM 340 E, Microm International GmbH, Waldoff, Germany) and relaxed in a $42^{\circ} \mathrm{C}$ water bath. For both slide sets, 2 to 3 serial sections from each experimental quarter were mounted to Superfrost Plus microscope slides (Thermo Fisher Scientific, Waltham, MA), drained, and dried 
at $37^{\circ} \mathrm{C}$ for $24 \mathrm{~h}$. All sections were deparaffinized in 3 changes of a xylene substitute (Clear-Rite 3, Thermo Fisher Scientific) and rehydrated in deionized water using a graded ethanol series before being subjected to different immunohistochemistry protocols.

Cleaved Caspase-3 Staining. The cleaved caspase-3 slide set was prepared using a bright-field immunohistochemistry kit (Histostain-Plus IHC Kit, Thermo Fisher Scientific). Antigens were retrieved by immersing slides in $10 \mathrm{~m} M$ citrate buffer, $\mathrm{pH}$ 6.0, for 30 min at $95^{\circ} \mathrm{C}$. Slides were removed from the heat source after antigen retrieval and allowed to cool to room temperature while remaining submerged in citrate buffer. Sections were subsequently washed in $0.9 \% \mathrm{NaCl}$ PBS, $\mathrm{pH}$ 7.4, quenched using $3 \% \mathrm{H}_{2} \mathrm{O}_{2}$ for $10 \mathrm{~min}$ at room temperature, and washed again with PBS. A hydrophobic barrier pen (Liquid Blocker, Daido Sangyo Co. Ltd., Saitama, Japan) was used to separate tissue sections on the same slide to isolate section-specific reagents and antibody mixtures. All sections were blocked using the provided kit reagent for $30 \mathrm{~min}$.

The blocking agent was aspirated from all sections and $100 \mu \mathrm{L}$ of the blocking reagent was reapplied to a single section on each slide; this section served as the negative control. Remaining sections received 100 $\mu \mathrm{L}$ of the primary antibody mixture that contained the cleaved caspase- 3 antibody (Table 1) suspended in the kit-provided blocking solution and incubated in a dark humidified chamber at $4^{\circ} \mathrm{C}$ for $16 \mathrm{~h}$. After primary antibody incubation, solutions were aspirated and sections were washed thrice with PBS for 2 min at each washing. All tissue sections were then incubated with $100 \mu \mathrm{L}$ of the kit-provided streptavidin-conjugated horseradish peroxidase for $10 \mathrm{~min}$ at room temperature before being washed thrice more with PBS for 2 min at each washing. The horseradish peroxidase streptavidin conjugate was reacted with freshly prepared DAB (3,3'-diaminobenzidene) chromogen for 3 min and immediately washed 3 times with PBS for 1 min at each washing. Sections were lightly counterstained by quickly dipping slides in hematoxylin 3 times and rins- ing with tap water. Sections were dehydrated, dried, and coverslipped (Permount, Thermo Fisher Scientific).

$K \boldsymbol{i}-67$ Staining. Antigen retrieval and isolation of tissue sections for the Ki-67 slide set followed the procedures described for the cleave caspase- 3 slide set with minor modification. Differing from the previous slide set, the Ki-67 slide set sections were blocked using a universal blocking agent (CAS Block, Thermo Fisher Scientific) for $30 \mathrm{~min}$.

The CAS Block was aspirated from the sections and $100 \mu \mathrm{L}$ of CAS Block was reapplied to a single negative control section on each slide. The remaining sections received $100 \mu \mathrm{L}$ of the primary antibody mixture containing Ki-67 antibody (Table 1) suspended in CAS Block. Sections were incubated in a dark humidified chamber at $4^{\circ} \mathrm{C}$ for $16 \mathrm{~h}$. After primary antibody incubation, solutions were aspirated and sections were washed thrice with PBS for $5 \mathrm{~min}$ at each washing. All tissue sections were then incubated with $100 \mu \mathrm{L}$ of a secondary antibody mixture, containing the secondary antibody (Table 1) suspended in CAS Block, at room temperature for $60 \mathrm{~min}$. Secondary antibody mixtures were aspirated and sections were washed again with PBS. Residual hydrophobic barrier pen outlines were removed and sections were coverslipped using ProLong Gold Antifade Mountant containing DAPI (Thermo Fisher Scientific). Coverslipping mountant was cured overnight before sections were imaged.

\section{Imaging and Image Analysis}

Cleaved Caspase-3 Sections. For the cleaved caspase-3 stained sections, 6 images of mammary parenchyma were randomly identified and imaged. Images were acquired with a bright field color camera (Retiga R6, QImaging Corporation, Surrey, BC, Canada) affixed to an Olympus BX43 light microscope (Olympus Corporation of the Americas, Center Valley, PA) with a $40 \times$ objective. With the microscope configuration used, the total area imaged in 6 microscopic fields was 0.458 $\mathrm{mm}^{2}$.

Table 1. Antibodies, manufacturer, catalog numbers, and dilutions employed to stain cleaved caspase-3 and Ki-67-positive nuclei in mammary tissues

\begin{tabular}{lllll}
\hline Antibody & Antibody type & Manufacturer & Catalog number & Dilution \\
\hline Cleaved caspase-3 & $1^{\circ}$ & Cell Signaling & 9579 & $1: 200$ \\
Ki-67 & $1^{\circ}$ & Thermo Fisher Scientific & \\
Alexa Fluor 594 & $2^{\circ}$ & Thermo Fisher Scientific & 9106 & $1: 200$ \\
\hline
\end{tabular}

${ }^{1}$ Cell Signaling Technology, Danvers, MA.

${ }^{2}$ Thermo Fisher Scientific, Waltham, MA. 
Acquired images were first analyzed by counting the number of positively stained cleaved caspase- 3 nuclei within the mammary epithelial and luminal compartments. Cells classified as epithelial included both mammary epithelial cells and myoepithelial cells. Any cell present in the gland lumen positively stained for cleaved caspase-3 was included in the cell counts; hence, both immune and epithelial cells would have been included in this count total. The respective compartmental counts were summed across the 6 randomly acquired images from each experimental quarter to produce a total number of positive cells across a constant area examined. This approach is similar to that previously described by Long et al. (2001). For each experimental quarter, a subset of 3 randomly selected images were examined once more so that cells within the intralobular stroma compartments could be differentiated and classified as being positive or negative for cleaved caspase-3 staining. During this examination, endothelial cells were avoided and 100 cells in each image were randomly identified and putatively differentiated as being either fibroblasts or immune cells by a single classifier. In addition, differentiated cells were simultaneously classified as being either positively or negatively stained for cleaved caspase-3. This approach resulted in 300 cells being differentiated, and these data were used to calculate the percentages for each cell type for each experimental quarter.

Ki-67-Stained Sections. For the Ki-67-stained sections, 8 mammary parenchyma fields of view were randomly identified and imaged for each experimental quarter tissue section. Regions of mammary parenchyma were identified using DAPI nuclear staining as a morphological index. Images were acquired using a Nuance FX multispectral imaging system (Perkin Elmer, Waltham, MA) affixed to a Nikon Eclipse E600 epifluorescence microscope (Nikon Instruments Inc., Melville, NY) with a Plan Fluor $40 \times$ objective. A mercury lamp was used as the excitation light source, and filter cubes, fitted with long pass emission filters, were used to excite specific secondary antibody and DAPI fluorophores. The Nuance system was configured to use tailored emission spectra to visualize DAPI-counterstained nuclei and positively stained Ki-67 nuclei. The product of this approach was 2 images for each field of view: 1 image containing DAPI-counterstained nuclei and 1 image containing Ki-67-stained nuclei. Correspondingly, 576 images were produced from the 288 imaged fields of view. A total area of $0.718 \mathrm{~mm}^{2}$ was imaged for each experimental quarter $(8$ microscopic fields of view) using the configured microscope.

Acquired images were analyzed by counting the number of positively stained Ki-67 nuclei within the mammary epithelial and stromal compartments in each image. The respective compartmental counts completed for each image were summed across the 8 random images acquired from each experimental quarter, as described earlier, to produce a total number of positive cells across a constant area examined.

\section{Statistical Analysis}

Cleaved Caspase-3 Nuclei. The total number of positively stained cleaved caspase- 3 nuclei in the epithelial and luminal compartments, quantified across the 6 images per experimental quarter, were analyzed using the MIXED procedure in SAS 9.4 (SAS Institute Inc., Cary, NC). Total epithelial and luminal cleaved caspase-3 cell counts served as the dependent variable in separate models. The fixed independent effects of quarter treatment $(\mathrm{n}=2)$ and day euthanized $(\mathrm{n}=$ 2 ) were included in both models; their interaction was included if $P \leq 0.10$. Cow nested within day of euthanasia was specified as a random effect in both models. The positive cleaved caspase- 3 nuclei counts in the luminal compartment were non-normally distributed and yielded non-normally distributed residuals when tested against the model's effects. In response, nuclei counts were $\log _{10}$-transformed and analyzed to satisfy the assumptions of normal distribution and equal variance. All presented least squares means were estimated using untransformed nuclei counts, but the $P$-values produced by the $\log _{10}$-transformed luminal nuclei count model are reported.

Differential cleaved caspase-3 intralobular stroma cell counts were also analyzed using the MIXED procedure. Each cell type percentage served as the dependent variable in separate models. Models were identical and included the fixed independent effects of quarter treatment $(\mathrm{n}=2)$ and day euthanized $(\mathrm{n}=2)$ and their interaction if $P \leq 0.10$. Cow nested within day of euthanasia was specified as a random effect in all models. Resultant least squares means were contrasted using Fisher's least significant differences test.

Ki-67 Nuclei. The summed number of Ki-67stained nuclei in the epithelial and stromal compartments were analyzed using the MIXED procedure as the dependent variable in separate models. The models' effects were identical to that used to test cleaved caspase nuclei counts. The positive Ki-67 nuclei counts in the stroma compartment were non-normally distributed and yielded non-normally distributed residuals when tested against the model's effects; these counts were $\log _{10}$-transformed and analyzed as described earlier. Again, the presented least squares means for epithelial and stromal Ki-67 nuclei counts were calculated using 
untransformed nuclei counts, but $P$-values yielded by the $\log _{10}$-transformed stroma nuclei count model are reported.

\section{RESULTS}

\section{Cleaved Caspase-3 Positive Nuclei}

Representative images of cleaved caspase- 3 staining are presented in Figure 1, and the summed cleaved caspase-3 positive nuclei least squares means counts recorded for the epithelial compartment are summarized and contrasted in Table 2. Cleaved caspase-3 nuclei counts in the epithelial compartment tended to be higher in challenged quarters than saline-infused quarters (1.6 vs. $2.6 \pm 0.4$ nuclei; $P=0.060)$, but nuclei counts were not affected by day of euthanasia $(P=0.29)$. Positive cleaved caspase- 3 counts in the epithelial compartment were not influenced by the effect of quarter treatment interacting with euthanasia day $(P=0.31)$.

Summed cleaved caspase-3 positive nuclei counts for the luminal compartment are also presented and contrasted in Table 2. The number of cleaved caspase-3 positive nuclei in challenged epithelial lumens was 10fold greater than that observed in saline quarters $(2.0$ vs. $0.2 \pm 0.5$ nuclei; $P=0.002$ ). Neither the effect of euthanasia day nor the effect of euthanasia day interacting with quarter treatment affected the number of cleaved caspase-3 positive nuclei in epithelial lumens $(P$ $=0.52$ and 0.19 , respectively).

Overall, a greater percentage of positively stained cleaved caspase-3 nuclei in the intralobular stroma compartment were present in saline-infused quarters than challenged quarters (45.9 vs. $36.9 \pm 2.1 ; P<$ 0.0001); however, the percentage of positively stained cells did not differ between d 5 or 10 of euthanasia (41.1 vs. $41.7 \pm 2.8$, respectively; $P=0.88$ ). The differential intralobular stroma cell type percentages for cells staining positive and negative for cleaved caspase-3 are presented and contrasted in Table 3. A greater percentage of positively stained fibroblasts were observed in salineinfused quarters than Staph. aureus quarters (24.7 vs. $15.7 \pm 1.2 ; P<0.0001)$. Conversely, saline quarters displayed a lower percentage of immune cells staining positive for cleaved capase-3 than challenged quarters $(27.8$ vs. $37.0 \pm 1.5 ; P<0.0001)$. Saline-infused quarters did not differ from Staph. aureus-challenged quarters in the percentage of fibroblasts not stained for cleaved caspase- 3 or the percentage of immune cells positive for cleaved caspase- 3 expression $(P=0.77$ and 0.98 , respectively). Furthermore, the effect of euthanasia day and the effect of euthanasia day interacting with treatment did not significantly affect any of the cells staining either positive or negative for cleaved capsase-3 $(P \geq 0.37)$.

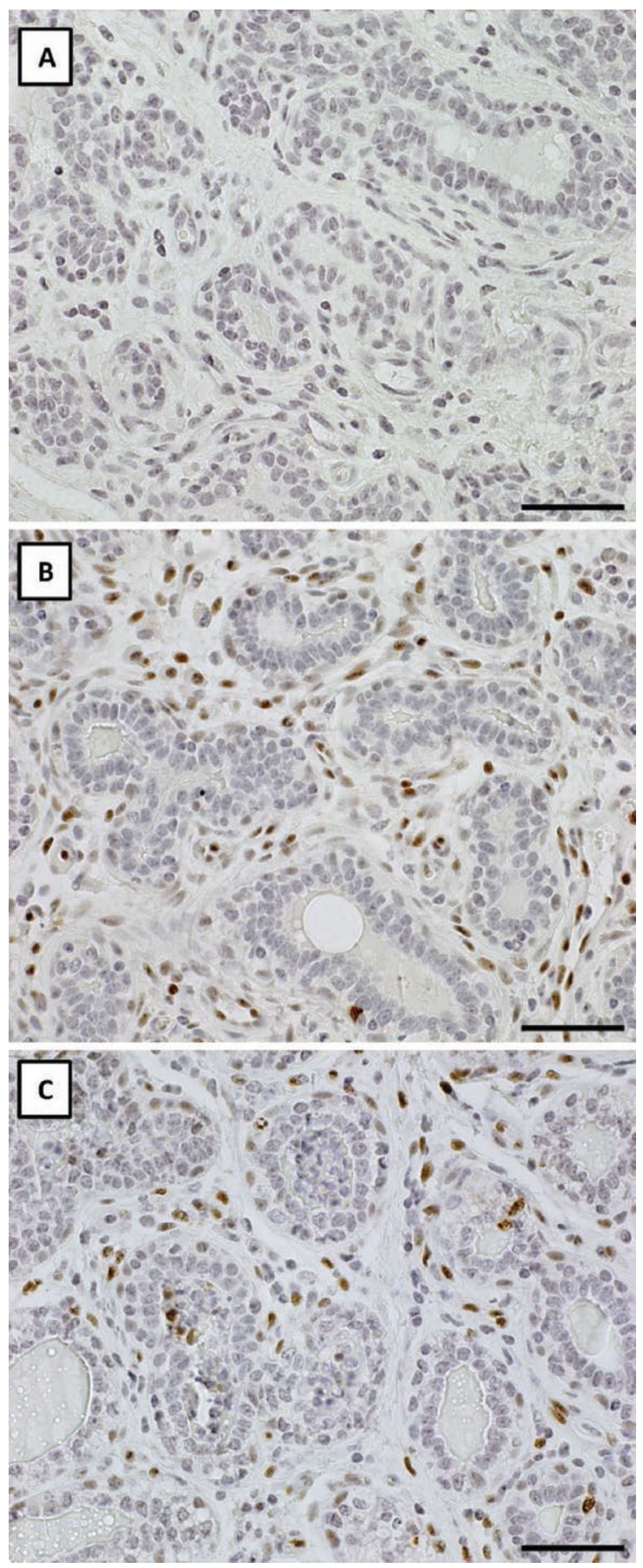

Figure 1. Staining of cleaved caspase-3-positive nuclei in mammary tissues from saline- and Staphylococcus aureus-infused glands. Panel A depicts a negative control section where the primary cleaved caspase-3 antibody was omitted; sections were lightly counterstained with hematoxylin. Panel B depicts tissue from a saline-infused quarter with extensive staining for cleaved caspase-3 (brown nuclei) for nuclei in the intralobular stroma compartment, but limited staining of mammary epithelial nuclei. Panel C displays tissues from a Staph. aureus-infused gland with several cleaved caspase-3-positive nuclei in the luminal compartment as well as in the mammary epithelium. A reduced percentage of nuclei in the intralobular stroma compartment is also evident. Magnification is the same for all images and scale bars $=50 \mu \mathrm{m}$. 
Table 2. Least squares means counts of positive cleaved caspase-3 nuclei in mammary epithelial and luminal compartments $^{1}$

\begin{tabular}{|c|c|c|c|c|c|c|c|c|}
\hline \multirow[b]{2}{*}{ Item } & \multicolumn{4}{|c|}{ Treatment } & \multicolumn{4}{|c|}{ Euthanasia day } \\
\hline & Saline & Challenge & SEM & $P$-value & d 5 & d 10 & SEM & $P$-value \\
\hline Epithelium & 1.6 & 2.6 & 0.4 & 0.06 & 1.7 & 2.5 & 0.5 & 0.29 \\
\hline Lumen & 0.2 & 2.0 & 0.5 & $<0.01$ & 1.3 & 0.8 & 0.5 & 0.52 \\
\hline
\end{tabular}

${ }^{1}$ Data expressed as number of positive cells observed across 6 parenchymal tissue fields of view $\left(0.458 \mathrm{~mm}^{2}\right)$.

\section{Ki-67-Positive Nuclei}

Representative images of Ki-67 staining are presented in Figure 2, and summed Ki-67 nuclei least squares means counts for the epithelial compartment are summarized and contrasted in Table 4. Total Ki67-positive nuclei counts in the epithelial compartment of saline-infused quarters were not different from those in challenged quarters $(P=0.97)$ and were not different between mammary tissues collected 5 or $10 \mathrm{~d}$ postchallenge $(P=0.78)$. The effect of quarter treatment interacting with euthanasia day did not affect positive Ki-67 nuclei counts in the epithelial compartment $(P$ $=0.98)$.

Summed Ki-67 nuclei least squares means counts for the intralobular stroma compartment are also summarized and contrasted in Table 4 . The number of Ki-67positive nuclei in the intralobular stroma compartment was 2.1-fold higher in challenged quarters than salineinfused quarters ( 38.1 vs. $18.0 \pm 5.6$ nuclei; $P=0.020$ ). The effects of euthanasia day and the interaction of euthanasia day and quarter treatment did not influence positive Ki-67 nuclei counts in the intralobular stroma compartment ( $P=0.27$ and 0.36 , respectively).

\section{DISCUSSION}

A chief objective of our study was to examine apoptosis and proliferation of mammary epithelial cells in uninfected and Staph. aureus-infected mammary glands that were stimulated to rapidly grow and develop using an induced mammary growth protocol. As expected, Staph. aureus-infected mammary tissues tended to have a greater number of mammary epithelial cells staining positive for the apoptosis marker, cleaved caspase-3, than uninfected quarters. This observation is consistent with that of Long et al. (2001) and Andreotti et al. (2017), who both reported a greater number of mammary epithelial cells undergoing apoptosis in mastitic quarters than uninfected quarters. Differing from these previous reports was the observation that Staph. aureus- and saline-infused quarters had a similar number of proliferating epithelial cells; Long et al. (2001) and Andreotti et al. (2017) both reported a greater number of proliferating epithelial cells in mastitic quarters. This discrepancy is believed to be a function of the different physiological states of mammary tissues being examined. As discussed, the tissues examined here were stimulated to rapidly grow and develop, whereas the mammary tissues examined by Long et al. (2001) and Andreotti et al. (2017) were from lactating and actively involuting mammary glands, respectively; limited mammary growth is expected to occur during these physiological states. Others have reviewed (Leoni et al., 2015; Landén et al., 2016) how tissue insults activate wound repair mechanisms that promote cellular proliferation in the affected tissues and drive tissue remodeling and healing. We suspected that any wound repair mechanisms that would have been activated in the Staph. aureus-infused quarters would not have had an additive effect in increasing epithelial proliferation because epithelial proliferation had already been upregulated via the estradiol and progesterone treatment; accordingly, no difference would be observed. Regardless, when these mammary tissues were examined previously (Enger et al., 2018), less mammary epithelium was

Table 3. Least squares means percentages ${ }^{1}$ of fibroblast and immune cell types in the intralobular stromal compartment staining positive $(+)$ or negative $(-)$ for cleaved caspase-3

\begin{tabular}{|c|c|c|c|c|c|c|c|c|}
\hline \multirow[b]{2}{*}{ Item } & \multicolumn{4}{|c|}{ Treatment } & \multicolumn{4}{|c|}{ Euthanasia day } \\
\hline & Saline & Challenge & SEM & $P$-value & d 5 & d 10 & SEM & $P$-value \\
\hline Fibroblast + & 24.7 & 15.7 & 1.2 & $<0.01$ & 20.1 & 20.3 & 1.4 & 0.91 \\
\hline Fibroblast - & 26.7 & 26.4 & 1.4 & 0.77 & 27.4 & 25.7 & 1.7 & 0.51 \\
\hline Immune cell + & 21.2 & 21.2 & 1.5 & 0.98 & 21.0 & 21.4 & 2.0 & 0.90 \\
\hline Immune cell - & 27.8 & 37.0 & 1.5 & $<0.01$ & 32.0 & 32.8 & 1.9 & 0.77 \\
\hline
\end{tabular}

${ }^{1}$ Data expressed as the percentage of cells differentiated from 3 tissue fields of view (100 cells/field of view). 

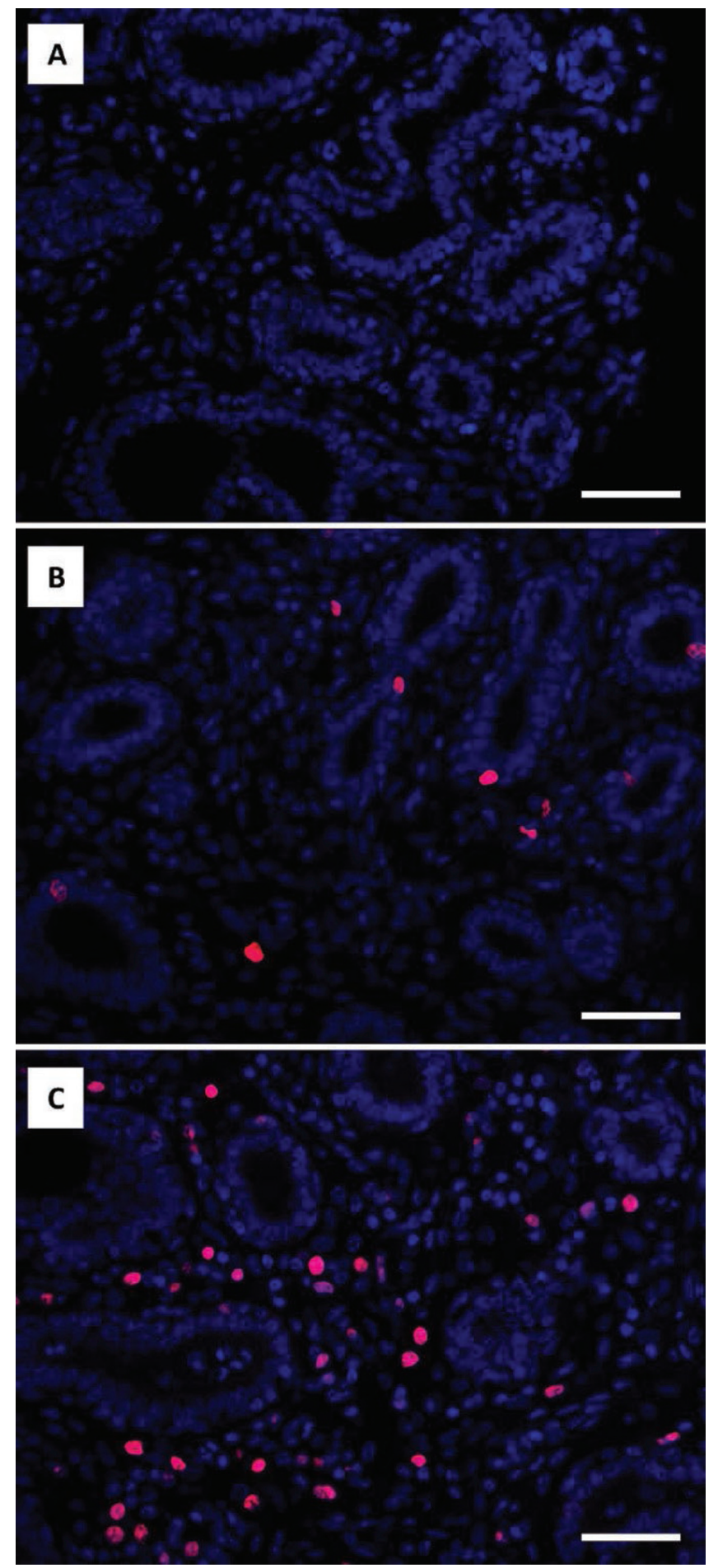

Figure 2. Staining of Ki-67-positive nuclei in mammary tissues from saline- and Staphylococcus aureus-infused glands. Panel A depicts a negative control section where the primary antibody (Ki-67) was omitted; nuclei are counterstained with DAPI (blue; Thermo Fisher Scientific, Waltham, MA). Panel B depicts tissue from a saline quarter with few intralobular stroma nuclei staining positive for Ki-67 (red). Panel C portrays mammary tissues from a Staph. aureus-challenged gland containing a significant number of Ki-67-positive cells in the intralobular stroma compartment. Magnification is the same for all images and scale bars $=50 \mu \mathrm{m}$. observed in challenged quarters, and this observation is associated with the increased epithelial apoptosis described here. Overall, an impairment in the growth of mammary epithelium is indicated.

Another key objective of our evaluation was to characterize the occurrence of cellular proliferation in the intralobular stromal compartment of the saline- and Staph. aureus-infused glands. A greater number of proliferating cells were present in the intralobular stromal compartment of Staph. aureus challenge quarters than saline-infused quarters, which was expected and agrees with previous reports (Long et al., 2001; Andreotti et al., 2017). An increase in the number of proliferating cells in the intralobular stromal compartment would be expected to contribute to the previously observed larger areas of intralobular stroma in Staph. aureus-infused quarters (Enger et al., 2018). Other investigators have also described increased stromal tissue areas in infected quarters relative to uninfected quarters for both lactating (Spencer, 1949; Akers and Thompson, 1987) and nonlactating glands (Sordillo et al., 1989; Trinidad et al., 1990; Andreotti et al., 2014), and the increased cellular proliferation of cells in the intralobular stromal compartment may, in part, contribute to the reported increased areas of intralobular stroma. Nonetheless, the heterogeneous cell population comprising the intralobular stroma compartment should be considered for an appreciation of the cellular and tissue changes occurring as a result of Staph. aureus challenge. This could not be accomplished using the florescent microscopy approach used here, as limited cellular morphology is present in florescent imaging and limited cellular morphological features were present to differentiate cells. Regardless, the increased proliferation observed in the intralobular stromal compartment of Staph. aureus-challenged glands is believed to be the result of an increase in the number of proliferating fibroblasts, in response to wound repair mechanisms, and an increase in the number of proliferating immune cells undergoing selection and expansion. These immune cells would be expressing proliferation- or survival-associated proteins.

Overall, Staph. aureus-challenged mammary tissues contained a lower percentage of apoptotic cells than saline-infused quarter tissues, which further coincides with the greater areas of intralobular stroma in Staph. aureus quarters relative to saline. A novel facet of this investigation was the putative differentiation of intralobular stromal cells staining positive and negative for cleaved caspase-3. We observed that a greater percentage of nonapoptotic immune cells were present in Staph. aureus- than saline-infused quarters. This was not surprising, as the increased infiltration of immune cells into the examined mammary tissues would increase the proportion of immune cells in the stroma, which 
Table 4. Least squares means counts of positive Ki-67 nuclei in mammary epithelial and stromal compartments ${ }^{1}$

\begin{tabular}{lccccccccc}
\hline & \multicolumn{4}{c}{ Treatment } & & \multicolumn{4}{c}{ Euthanasia day } \\
\cline { 2 - 3 } \cline { 8 - 10 } Item & Saline & Challenge & SEM & $P$-value & & $\mathrm{d} 5$ & $\mathrm{~d} 10$ & $\mathrm{SEM}$ & $P$-value \\
\hline Epithelium & 32.1 & 32.2 & 4.7 & 0.97 & & 33.3 & 30.9 & 6.2 & 0.78 \\
Stroma & 18.0 & 38.1 & 5.6 & 0.02 & & 31.9 & 24.3 & 5.8 & 0.27 \\
\hline
\end{tabular}

${ }^{1}$ Data expressed as number of positive cells observed across 8 parenchymal tissue fields of view $\left(0.718 \mathrm{~mm}^{2}\right)$.

have infiltrated in response to IMI. More interestingly, a greater percentage of nonapoptotic fibroblasts were present in Staph. aureus- than saline-infused quarters. The consequence of fewer apoptotic fibroblasts in the Staph. aureus-infected glands may contribute to the accumulation of connective tissues in the gland and result in the continued presence of intralobular stromal tissue in these glands. Andreotti et al. (2014) previously described increased amounts of type 1 collagen in actively involuting, chronically Staph. aureus-infected quarters relative to uninfected quarters. In the context of the present experiment, Howe et al. (1975) and Croom et al. (1976) examined mammary tissues from nonpregnant, nonlactating animals that were subject to similar hormonal induction protocols. Those researchers observed a continued regression of intralobular stroma and expansion of luminal space when the collected mammary tissues were examined throughout the progression of the induction protocol. When these reports are considered with the results of the present study, it indicates that the intralobular stromal compartment is failing to regress or is actively expanding in the Staph. aureus-challenged glands, which would not allow for the expansion of the luminal space. Either way, an impairment in mammary development is indicated.

At first examination of these tissues, the effect of euthanasia day did not influence any of the measures examined. As previously discussed by Enger et al. (2018), it may be that examining tissues at $5 \mathrm{~d}$ postchallenge was too late to capture the temporal changes occurring in the mammary tissues resulting from the Staph. aureus challenge.

\section{CONCLUSIONS}

The results of this study showed that Staph. aureus challenge of rapidly growing, nonlactating mammary glands increased the amount of mammary epithelial cells undergoing apoptosis but did not affect the number of proliferating mammary epithelial cells. Furthermore, a greater number of proliferating cells were present in the intralobular stromal compartment of Staph. aureuschallenged quarters than saline-infused quarters, which was accompanied by a lower percentage of apoptotic cells present in the intralobular stromal compartment of Staph. aureus glands. When these results were considered with the results from our previous examination of these tissues (Enger et al., 2018), we found that IMI in rapidly growing nonlactating mammary glands limits gland growth by differentially affecting cellular apoptosis and proliferation in affected tissues, which is expected to impair future milk yield and reduce productive herd life.

\section{ACKNOWLEDGMENTS}

This work was supported by a USDA-NIFA-AFRI (Washington, DC) competitive predoctoral fellowship (2017-67011-26049), awarded to B. D. Enger, a Virginia Agricultural Council (Richmond, VA) grant (VAC Project no. 685) awarded to R. M. Akers, and Professorship funds (Horace E. and Elizabeth F. Alphin Professorship, College of Agriculture and Life Sciences, Virginia Tech, Blacksburg) awarded to R. M. Akers.

\section{REFERENCES}

Akers, R. M., and S. C. Nickerson. 2011. Mastitis and its impact on structure and function in the ruminant mammary gland. J. Mammary Gland Biol. Neoplasia 16:275-289.

Akers, R. M., and W. Thompson. 1987. Effect of induced leucocyte migration on mammary cell morphology and milk component biosynthesis. J. Dairy Sci. 70:1685-1695.

Andreotti, C. S., E. A. Pereyra, C. Baravalle, M. S. Renna, H. H. Ortega, L. F. Calvinho, and B. E. Dallard. 2014. Staphylococcus aureus chronic intramammary infection modifies protein expression of transforming growth factor beta (TGF-beta) subfamily components during active involution. Res. Vet. Sci. 96:5-14.

Andreotti, C. S., E. A. L. Pereyra, S. C. Sacco, C. Baravalle, M. S. Renna, H. H. Ortega, L. F. Calvinho, and B. E. Dallard. 2017. Proliferation-apoptosis balance in Staphylococcus aureus chronically infected bovine mammary glands during involution. J. Dairy Res. 84:181-189.

Bayles, K. W., C. A. Wesson, L. E. Liou, L. K. Fox, G. A. Bohach, and W. R. Trumble. 1998. Intracellular Staphylococcus aureus escapes the endosome and induces apoptosis in epithelial cells. Infect. Immun. 66:336-342.

Capuco, A. V., R. M. Akers, and J. J. Smith. 1997. Mammary growth in Holstein cows during the dry period: Quantification of nucleic acids and histology. J. Dairy Sci. 80:477-487.

Croom, W. J., R. J. Collier, D. E. Bauman, and R. L. Hays. 1976. Cellular studies of mammary tissue from cows hormonally induced into lactation: Histology and ultrastructure. J. Dairy Sci. 59:12321246.

Eberhart, R. J. 1986. Management of dry cows to reduce mastitis. J. Dairy Sci. 69:1721-1732. 
Enger, B. D., C. E. Crutchfield, T. T. Yohe, K. M. Enger, S. C. Nickerson, C. L. M. Parsons, and R. M. Akers. 2018. Staphylococcus aureus intramammary challenge in non-lactating mammary glands stimulated to rapidly grow and develop with estradiol and progesterone. Vet. Res. 49:47.

Fox, L. K. 2009. Prevalence, incidence and risk factors of heifer mastitis. Vet. Microbiol. 134:82-88.

Hogan, J. S., E. A. Berry, J. E. Hillerton, H. Hogeveen, S. C. Nickerson, S. P. Oliver, G. M. Pighetti, P. Rapnicki, Y. H. Schukken, and L. K. Smith. 2016. Current Concepts of Bovine Mastitis. 5th ed. The National Mastitis Council, New Prague, MN.

Howe, J. E., C. W. Heald, and T. L. Bibb. 1975. Histology of induced bovine lactogenesis. J. Dairy Sci. 58:853-860.

Hu, Q., X. Cui, L. Tao, L. Xiu, T. Wang, and X. Wang. 2014. Staphylococcus aureus induces apoptosis in primary bovine mammary epithelial cells through Fas-FADD death receptor-linked caspase-8 signaling. DNA Cell Biol. 33:388-397.

Jones, G. M., R. E. Pearson, G. A. Clabaugh, and C. W. Heald. 1984. Relationships between somatic cell counts and milk production. J. Dairy Sci. 67:1823-1831.

Landén, N. X., D. Li, and M. Stahle. 2016. Transition from inflammation to proliferation: A critical step during wound healing. Cell. Mol. Life Sci. 73:3861-3885.

Leoni, G., P. A. Neumann, R. Sumagin, T. L. Denning, and A. Nusrat. 2015. Wound repair: Role of immune-epithelial interactions. Mucosal Immunol. 8:959-968.
Long, E., A. V. Capuco, D. L. Wood, T. Sonstegard, G. Tomita, M. J. Paape, and X. Zhao. 2001. Escherichia coli induces apoptosis and proliferation of mammary cells. Cell Death Differ. 8:808-816.

Nickerson, S. C. 1980. Histological and cytological response of the bovine mammary gland to experimental $S$. aureus infection. $\mathrm{PhD}$ dissertation. Department of Dairy Science, Virginia Polytechnic Institute and State University, Blacksburg.

Smith, T. H., L. K. Fox, and J. R. Middleton. 1998. Outbreak of mastitis caused by one strain of Staphyoloccus aureus in a closed dairy herd. J. Am. Vet. Med. Assoc. 212:553-556.

Sordillo, L. M., S. C. Nickerson, and R. M. Akers. 1989. Pathology of Staphylococcus aureus mastitis during lactogenesis: Relationships with bovine mammary structure and function. J. Dairy Sci. 72:228-240.

Spencer, G. R. 1949. The significance of hypersensitivity in bovine mastitis as determined by a study of its pathogenesis. PhD Diss. Department of Dairy Science, University of Wisconsin-Madison.

Trinidad, P., S. C. Nickerson, and R. W. Adkinson. 1990. Histopathology of staphylococcal mastitis in unbred dairy heifers. J. Dairy Sci. 73:639-647.

Tucker, H. A. 1987. Quantitative estimates of mammary growth during various physiological states: A review. J. Dairy Sci. 70:1958-1966. 